\title{
Study of CT-based positron range correction in high resolution 3D PET imaging
}

\author{
J. Cal-González ${ }^{\text {a,* }}$,J.L. Herraiz ${ }^{\text {a }}$, S. España ${ }^{\text {b }}$, E. Vicente ${ }^{\text {a,c }}$, E. Herranz ${ }^{\text {a }}$, M. Desco ${ }^{\text {d }}$,J.J. Vaquero ${ }^{\mathrm{e}}$, J.M. Udías ${ }^{\text {a }}$ \\ ${ }^{a}$ Grupo de Física Nuclear, Dpto. Física Atómica, Molecular y Nuclear, Universidad Complutense de Madrid, Spain \\ ${ }^{\mathrm{b}}$ Department of Radiation Oncology, Massachusetts General Hospital and Harvard Medical School, Boston, MA, USA \\ ${ }^{\mathrm{C}}$ Instituto de Estructura de la Materia, Consejo Superior de Investigaciones Científicas (CSIC), Madrid, Spain \\ ${ }^{\mathrm{d}}$ Unidad de Medicina y Cirugía Experimental, Hospital General Universitario Gregorio Marañón, Madrid, Spain \\ e Dpto. de Bioingeniería e Ingeniería Espacial, Universidad Carlos III, Madrid, Spain
}

\section{A R T I C L E I N F O}

\section{Available online 16 December 2010}

Keywords:

Positron emission tomography

Computed tomography

Positron range

Image quality

Monte Carlo simulation

\begin{abstract}
A B S T R A C T
Positron range limits the spatial resolution of PET images and has a different effect for different isotopes and positron propagation materials. Therefore it is important to consider it during image reconstruction, in order to obtain optimal image quality. Positron range distributions for most common isotopes used in PET in different materials were computed using the Monte Carlo simulations with PeneloPET. The range profiles were introduced into the 3D OSEM image reconstruction software FIRST and employed to blur the image either in the forward projection or in the forward and backward projection. The blurring introduced takes into account the different materials in which the positron propagates. Information on these materials may be obtained, for instance, from a segmentation of a CT image. The results of introducing positron blurring in both forward and backward projection operations was compared to using it only during forward projection. Further, the effect of different shapes of positron range profile in the quality of the reconstructed images with positron range correction was studied. For high positron energy isotopes, the reconstructed images show significant improvement in spatial resolution when positron range is taken into account during reconstruction, compared to reconstructions without positron range modeling.
\end{abstract}

\section{Introduction}

The range of positrons in tissue is an important limitation to the spatial resolution achievable in 3D PET [1]. Recent developments in the detector technology have reduced crystal size and now there are small animal PET scanners with near $1 \mathrm{~mm}$ spatial resolution, such as the ARGUS [2]. This resolution is comparable to the positron range of some commonly used isotopes, such as ${ }^{68} \mathrm{Ga}(\sim 2 \mathrm{~mm})$ or ${ }^{82} \mathrm{Rb}$ $(\sim 4 \mathrm{~mm})$ [3]. Positron range appears as a blurring of the recon structed image. Based on measured positron range functions, Derenzo [4] proposed a method to remove the blurring in the reconstructed images in FBP. Recently, new methods to remove positron range have been developed using MAP during reconstruction [5,6].

Positron range in water has been measured experimentally for several medically important isotopes [7]. Monte Carlo simulations may help in estimating positron range and its effect on image quality $[1,3]$. In a previous work [8], we simulated positron interactions and annihilation with the PeneloPET code [9].

In a non homogeneous media, the blurring due to positron range is spatially variant depending on the variations in absorber media, given a different blurring that depends on the properties of the local media in which the positron is propagated. These proper ties can be obtained from a CT image [10].

\footnotetext{
* Corresponding author. Tel.: +34913944 484; fax: +34913945 193.

E-mail address: jacobo@nuclear.fis.ucm.es (J. Cal-González).
}

In this work we account for the material dependent positron range by modeling its effects during the 3D OSEM reconstruction [11], taking into account the material in which the positron is emitted, obtained for instance from a CT image.

\section{Materials and methods}

\subsection{Positron range simulations}

We used PeneloPET for simulating positron range. PeneloPET may simulate positron trajectory and initial energy for each positron coming from the decay process. This leads to accurate results. We chose ${ }^{68} \mathrm{Ga}$ as the isotope for the tests, as it has a positron range large enough compared to the resolution of the scanner.

\subsection{Image reconstruction with material dependent positron range blurring}

Positron range correction can be introduced into the iterative image reconstruction in two different ways:

- Introducing the effect of positron range in the System Response Matrix (SRM). This can be done using a factored system model where different positron range blurrings can be used by means of a realistic simulation that includes all the main physical 
effects into account [11] or by obtaining the SRM from point source measurements [12].

- Using the positron range profiles obtained from Monte Carlo simulations as an additional blurring applied to the object. In this case, the SRM should not incorporate positron range effects.

This work shows the results of the second method, as it allows for adapting the blurring to the local properties of the object (electronic density, effective $Z$ ) in which positrons are emitted. The SRM was simulated with PeneloPET [9] without positron range effects but with considering all other physical effects like non colinearity or inter crystal scatter. Following a similar approach as in Ref. [13] the positron range corrected OSEM (PR OSEM) algo rithm used in this work reads:

$x_{j}^{\prime} \equiv x_{j} \frac{\sum_{i} C_{i j}\left(y_{i} / \sum_{j} C_{i j} \tilde{x}_{j}\right)}{\sum_{i} C_{i j}}$

where $\tilde{x}_{j}$ is the object blurred by positron range that it is forward projected. $\tilde{x}_{j}$ is obtained by a convolution of the initial object with a blurring function $\rho^{(j)}$ corresponding to the positron range profile of the isotope source present at voxel $j$ and the material in that voxel.

$\tilde{x}_{j} \equiv x_{j} \otimes \rho^{(j)} \equiv \frac{\sum_{h} x_{j-h} \rho_{h}^{(j)}}{\sum_{h} \rho_{h}^{(j)}}$.

As it can be noticed, a miss matched projector/backprojector pair is used in Eq. (1). Indeed, positron range blurring is only taken into account in the projection operation. This approach has been used in several works [14,15] where it is shown that good reconstructed images can be attained with full modeling of blurring effects during the projection step, whereas a simplified modeling is employed into the backprojector.

\subsection{PeneloPET simulations of several IQ phantom acquisitions}

In order to measure the effects of the positron range in the image quality, acquisitions of an IQ phantom according to NEMA protocol [16] filled with different isotopes have been simulated in this work. This phantom is made of polymethylmethacrylate (PMMA) with $50 \mathrm{~mm}$ length and $30 \mathrm{~mm}$ diameter. The main body of the phantom is composed of a fillable cylindrical chamber with $30 \mathrm{~mm}$ diameter and $30 \mathrm{~mm}$ length (uniform region). The remaining $20 \mathrm{~mm}$ of the total length is a solid body with 5 fillable rods of $1,2,3,4$ and $5 \mathrm{~mm}$ diameters (at $7 \mathrm{~mm}$ from the center). At the end of the uniform region there is one cold region chamber and other hot region chamber. These chambers are composed of hollow cylinders with $15 \mathrm{~mm}$ length and $10 \mathrm{~mm}$ external diameter.

In order to measure the quality of our reconstructed images, we compared the spatial resolution and recovery coefficient [17] (RC) variation against noise of the image. Spatial resolution has been estimated as the average FWHM of the Gaussian fits to derivatives of radials profiles across the 2, 3, 4 and $5 \mathrm{~mm}$ diameter line sources of the IQ phantom. RC has been calculated from the number of counts obtained in the reconstruction for a Region of Interest (ROI) drawn inside the $3 \mathrm{~mm}$ in diameter line source, divided by number of counts of a cylindrical ROI taken in the uniform region of the phantom. Finally, noise in the image has been estimated as the ratio of the standard deviation of counts in each voxel of a cylindrical ROI taken in the uniform region of the phantom, and the total counts in this ROI.

In order to measure the effect in the quality of the images of positron range in different materials, we have simulated the following phantom (bone water phantom): A cylinder of $5 \mathrm{~cm}$ of diameter and $5 \mathrm{~cm}$ length centered in the FOV, filled with water. It contains a $1 \mathrm{~cm}$ off centered rod of bone material, $1 \mathrm{~cm}$ diameter and the same length as the cylinder. Two line sources with low activity and $1 \mathrm{~mm}$ of diameter were simulated, placed at $(1,0$, $0) \mathrm{cm}$ inside water and at $(1,0,0) \mathrm{cm}$ inside bone, respectively. The reconstruction was performed with FIRST after 100 image updates ( 2 iterations of 50 subsets each of them).

The simulations are chosen to represent the ARGUS small animal PET scanner. The simulated acquisitions have been recon structed with FIRST, a 3D OSEM procedure [11], with and without positron range modeling. Reconstruction parameters were as follows:

- Reconstruction method: FIRST, based on 3D OSEM and a realistic system response matrix.

- Image updates: 200 (20 iterations of 10 subsets each of them). A high number of iterations is chosen in order to have many intermediate images to assess image quality evolution.

- Energy window: $400700 \mathrm{keV}$.

- Number of voxels: $175 \times 175 \times 61$ voxels.

- Voxel size: $0.389 \times 0.389 \times 0.775 \mathrm{~mm}^{3}$.

\section{Results and discussion}

\subsection{Results obtained with NEMA IQ phantom}

Figs. 1 and 2 show a comparison between the resolution and RC versus noise obtained for a reconstruction, including the positron blurring in both forward and backprojection operations (dark blue curves), and when positron range is only included during forward projection (green curves). We can see that the use of positron blurring during backprojection just smoothes out the recon structed image, and the appearance of high resolution details in the image is delayed to larger number of image updates. Other than this, the resolution versus noise curves are nearly identical, given approximately the same resolution and RC values for the same level of noise in both cases.

These figures also show a comparison between the results obtained with the 3D OSEM reconstruction, which takes into account the correct positron range blurring, i.e., the same range employed during the PeneloPET simulation and reconstruction with erroneous positron range profiles, corresponding to a maximum energy of positrons that is $\pm 50 \%$ of the one corresponding to the maximum energy of correct profile. These curves are shown in light blue and dark colors. This comparison would allow us to assess what is the accuracy needed in the positron blurring determination, as

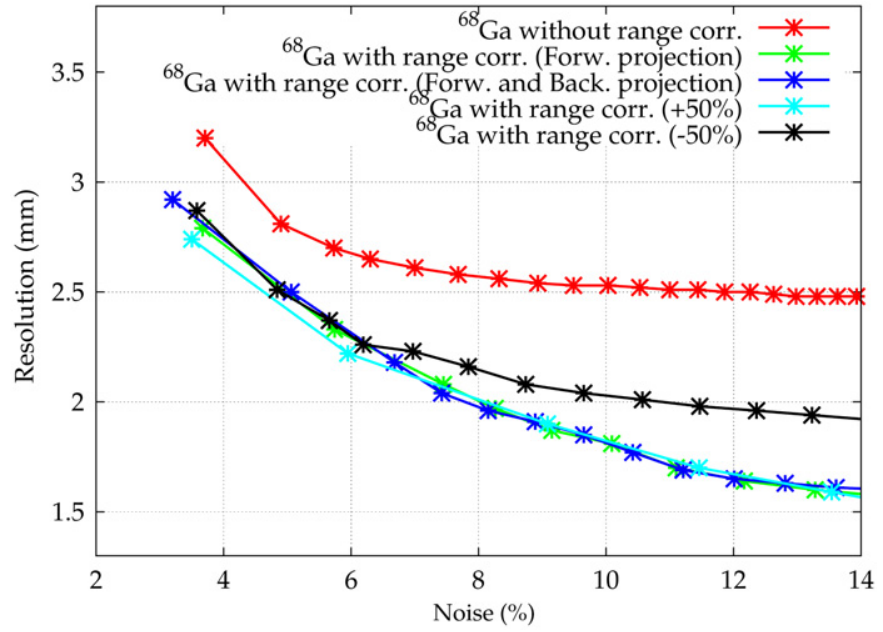

Fig. 1. Resolution-noise curves obtained for the IQ phantom filled with ${ }^{68} \mathrm{Ga}$ and reconstructed using 3D OSEM with and without range corrections. Each point corresponds to 10 image updates. 


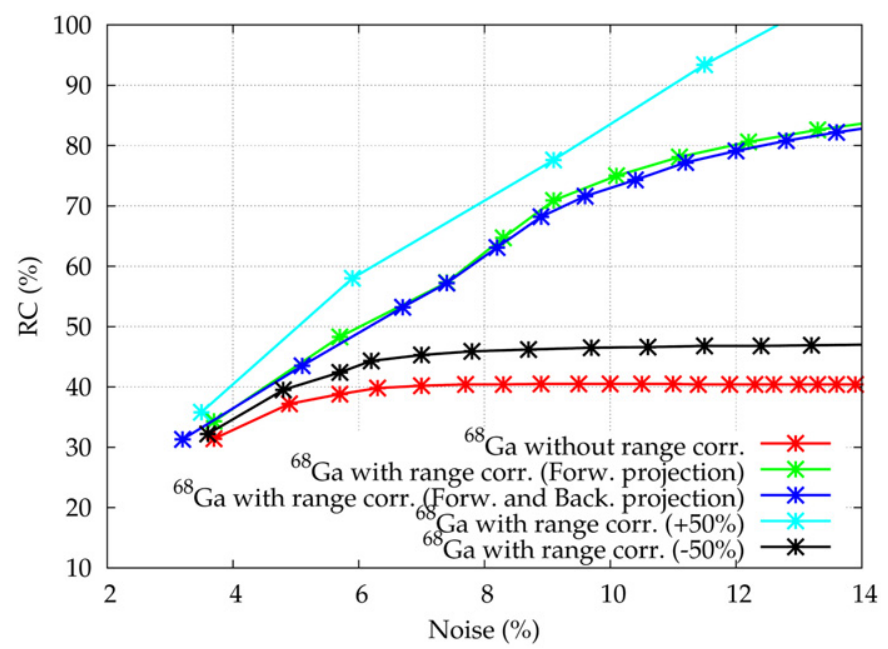

Fig. 2. RC-noise curves obtained for the $3 \mathrm{~mm}$ rod IQ phantom filled with ${ }^{68} \mathrm{Ga}$ and reconstructed using 3D OSEM with and without range corrections. Each point corresponds to 10 image updates.

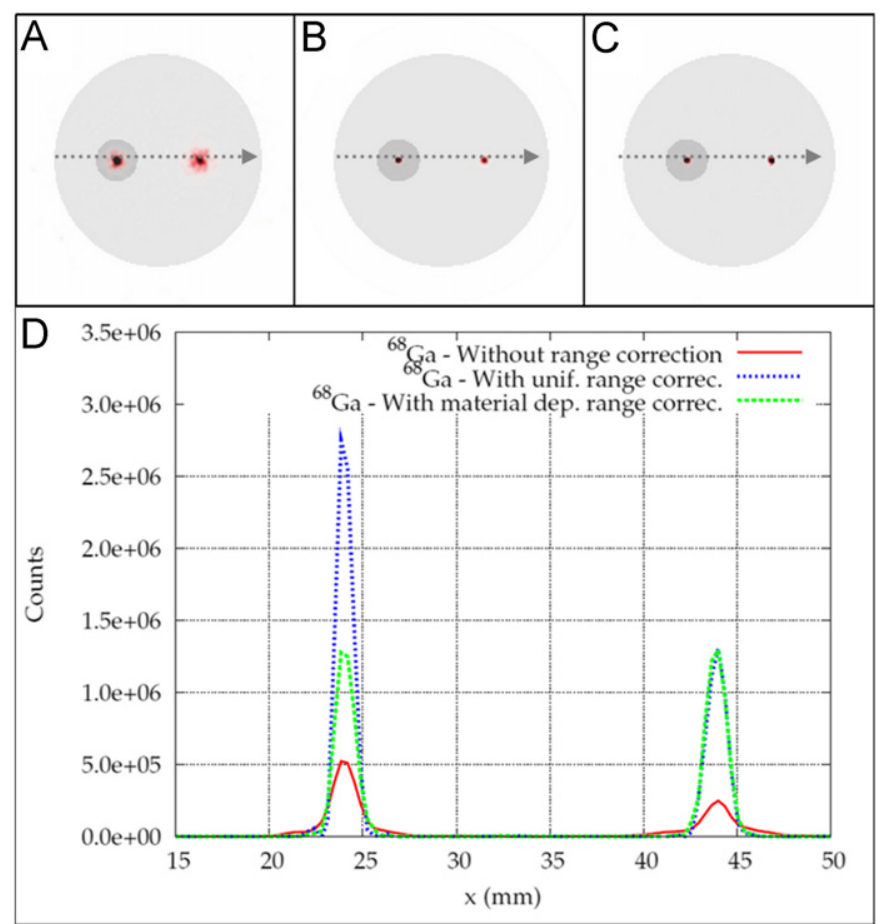

Fig. 3. Density map of simulated phantom along with the image of two ${ }^{68} \mathrm{Ga}$ line sources in bone and water after 3D OSEM reconstruction without range correction (A), with uniform range correction (B) and with material-dependent range correction (C). Images and profiles are compared at $10 \%$ noise. Panel D shows count line profiles along sources.

positron range has not been very well determined for high energy positron ranges.

The results obtained suggest that the use of erroneous positron range profiles with an error of $\pm 50 \%$ has a significant effect on the quality of the reconstructed images. Comparing images with the same noise level, under corrected images ( 50\% dark curves) have worst resolution and $\mathrm{RC}$ values than the curves corresponding to correct profiles (dark blue curves). On the other hand, although resolution and $\mathrm{RC}$ seem to be better in over corrected images (light blue curves) than in the correct ones (dark blue curves) inspection of the images shows that they have large ringing artifacts and produce very narrow line sources, narrower than the actual line sources. This is due to the over correction for range blurring.

Although it is not shown in these figures, we have also compared the effect of range corrections that are $\pm 10 \%$ of the ones obtained in the simulation and found that the effect on the noise resolution or RC curves is very similar to the ones where the correct positron range is employed. This means that for scanners with intrinsic resolution of the order of $1 \mathrm{~mm}$, positron range profiles should be known down to $0.10 .2 \mathrm{~mm}$.

\subsection{Material dependent positron range correction}

Fig. 3 shows the images reconstructed of the bone water phantom filled with ${ }^{68} \mathrm{Ga}$. In the first case, without range correction, the reconstructed size of the ${ }^{68} \mathrm{Ga}$ line sources in water is larger than in bone, which causes a visible difference in the respective heights of the activity profiles in bone and water. If we perform uniform positron range correction (taken the positron range profiles in water only) the line source placed in the bone region is over corrected, which shows a very high peak of the activity profiles. When material dependent positron range is modeled into the reconstruction, similar apparent sizes for the line sources and identical height of the activity profiles are obtained for annihila tions in the two materials.

\section{Conclusions}

- The approach proposed in this work improves significantly the quality of the reconstructed images, rendering range corrected images for large positron range isotopes, like ${ }^{68} \mathrm{Ga}$, practical and useful. For instance a spatial resolution down to around $1.8 \mathrm{~mm}$ can be obtained with range correction versus $2.5 \mathrm{~mm}$ without it.

- The use of positron blurring in the backprojection just causes the iterative algorithm to converge more slowly, but otherwise similar images are obtained.

- The use of erroneous positron range profiles ( $\pm 50 \%$ of error) has a significant effect on the quality of reconstructed images: infra corrected images with lower RC and resolution values or not valid over corrected images, with large ringing artifacts and too narrow line sources. On the other hand, for the scanner and isotope combination reviewed here, a $\pm 10 \%$ difference in the positron range employed for acquisition simulation and image reconstruction has a negligible effect on the reconstructed images.

- The use of material dependent positron range correction yields better image quality in heterogeneous medium, giving a similar apparent size and peak height of activity profiles for line sources in different media.

\section{Acknowledgment}

This work has been supported by the MEC (FPA2007 62216), the UCM (Grupos UCM, 910059), the CPAN (Consolider Ingenio 2010) CSPD 2007 00042, the RECAVA RETIC network, ARTEMIS S2009/ DPI 1802, the European Regional Development and ENTEPRASE grant, PSE 30000020095 and the Ministerio de Ciencia e Innova ción, Spanish Government.

\section{References}

[1] C.S. Levin, E.J. Hoffman, Phys. Med. Biol. 44 (1999) 781.

[2] Y. Wang, et al., J. Nucl. Med. 47 (11) (2006) 1891.

[3] C. Champion, C.L. Loirec, Phys. Med. Biol. 52 (2007) 6605.

[4] S.E. Derenzo, IEEE Trans. Nucl. Sci. NS33 (1) (1986) 565.

[5] B. Bai, R. Laforest, et al., IEEE NSS-MIC Conference Record, 2004, pp. 2501-2505. 
